\title{
Neck pounding during sinus rhythm: a new clinical manifestation of dual atrioventricular nodal pathways
}

\author{
P Geelen, J Primo, J Brugada, E Andries, P Brugada
}

\begin{abstract}
Objective-To determine the clinical and electrophysiological characteristics of patients with paroxysmal palpitations and neck pounding during sinus rhythm.

Methods-Clinical, electrocardiographic, and electrophysiological characteristics of six patients with paroxysmal palpitations and neck pounding during sinus rhythm were studied in basal conditions and when symptomatic. Response to treatment was observed.

Results-Baseline ECGs were normal (four patients) or had first degree atrioventricular block with intermittent $P R$ shortening. During symptoms, narrow QRS rhythms were seen without visible $P$ waves (three patients) or with $P$ waves partially hidden in the QRS complex (three patients). Dual atrioventricular nodal pathways were found in all five patients who had electrophysiological studies. In these patients the slow pathway conduction time was long enough (mean (SD), 425 (121) $\mathrm{ms}$ ) for ventricular activation after slow pathway conduction during sinus rhythm to coincide with the next atrial depolarisation, causing neck pounding during exercise (four patients) or at rest (two patients). Tachycardia was not induced in any patient. Medical treatment aggravated symptoms in three patients. A pacemaker was successfully used in two. Conclusions-Neck pounding during sinus rhythm is a clinical manifestation of dual atrioventricular nodal pathways. Medical treatment may aggravate symptoms but a pacemaker may offer definitive relief. (Heart 1998;79:490-492)
\end{abstract}

Keywords: neck pounding; nodal conduction; palpitations

Searching for specific symptoms is an important step in evaluating patients with palpitations. If the palpitations are accompanied by

12 January 1998

Table 1 Clinical characteristics of the patients

\begin{tabular}{lllllllll}
\hline Patient & Sex & $\begin{array}{l}\text { Age } \\
\text { (years) }\end{array}$ & $\begin{array}{l}\text { AH } \\
(\mathrm{ms})\end{array}$ & $\begin{array}{l}\mathrm{HV} \\
(\mathrm{ms})\end{array}$ & $\begin{array}{l}\text { AH slow } \\
(\mathrm{ms})\end{array}$ & $\begin{array}{l}\text { Retrograde } \\
\text { conduction }\end{array}$ & $\begin{array}{l}\text { Inducible } \\
\text { tachycardia }\end{array}$ & $\begin{array}{l}\text { Follow up } \\
\text { (months) }\end{array}$ \\
\hline 1 & $\mathrm{~F}$ & 24 & 85 & 40 & 360 & Poor & No & 48 \\
2 & $\mathrm{M}$ & 31 & 93 & 36 & 420 & None & No & 48 \\
3 & $\mathrm{M}$ & 36 & 100 & 43 & 400 & Poor & No & 24 \\
4 & $\mathrm{~F}$ & 41 & 315 & 45 & 315 & None & No & 6 \\
5 & $\mathrm{M}$ & 22 & 95 & 50 & 630 & None & No & 12 \\
6 & $\mathrm{~F}$ & 35 & No EPS & No EPS & No EPS & No EPS & No EPS & 34 \\
\hline
\end{tabular}

$\mathrm{AH}$, atrium-His interval; $\mathrm{AH}$ slow, shortest atrium-His interval during slow pathway conduction; EPS, electrophysiological study; HV, His-ventricle interval. pounding in the neck, this can be an important diagnostic clue. Slow irregular neck pounding (cannon waves) can occur in patients with ventricular tachycardia and atrioventricular dissociation. ${ }^{1}$ Our group has shown that rapid regular pounding in the neck is common during atrioventricular nodal reentrant tachycardia, owing to the almost simultaneous contraction of the atria and the ventricles during tachycardia. ${ }^{2}$ Thus regular pounding in the neck during tachycardia is a useful clinical marker of atrioventricular nodal reentrant tachycardia.

In this report we describe six patients with paroxysmal palpitations and prominent neck pounding caused by dual atrioventricular nodal pathways with specific electrophysiological properties. None of these patients, however, suffered from atrioventricular nodal reentrant tachycardias.

\section{The patients}

Between 1992 and 1997 we saw six patients with neck pounding during sinus rhythm who had dual atrioventricular nodal pathways with specific electrophysiological characteristics. Three were male and three female. All patients were young, with a mean (SD) age of 32 (7) years. Apart from paroxysmal palpitations they were free from any other complaints or diseases.

The characteristics of the patients are summarised in table 1.

\section{CLINICAL PRESENTATION}

Four patients presented with paroxysmal palpitations occurring with exercise and emotion. In two, palpitations occurred mostly at rest. The complaints had been present for many years in all the patients. The palpitations were described as an unpleasant awareness of the heartbeat in the chest, but were particularly felt in the neck. In all patients regular pulsations of the external jugular veins during the attacks were seen by family members, the attending physician, or the patients themselves. The attacks were generally accompanied by anxiety. No patient had ever suffered a syncope or complained of dizziness.

\section{ELECTROCARDIOGRAPHIC PRESENTATION}

Except during attacks of palpitations, the 12 lead electrocardiogram was completely normal in four patients. First degree atrioventricular block was present in two; intermittent shortening of the PR interval and PR interval alternans was also observed in these patients (fig 1). 


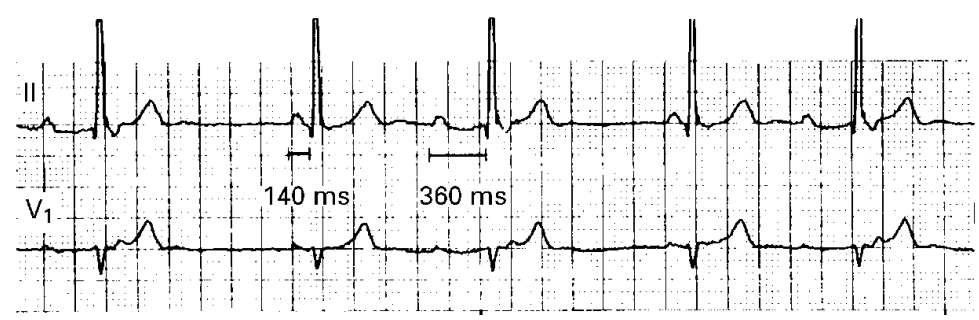

Figure 1 Lead II and V1 in patient 4 showing PR interval alternans caused by alternating atrioventricular conduction over the slow atrioventricular pathway (PR interval $360 \mathrm{~ms}$ ) and fast atrioventricular pathway (PR interval $140 \mathrm{~ms}$ ). Paper speed $25 \mathrm{~mm} / \mathrm{s}$.

During the attacks of palpitations and neck pounding, narrow QRS rhythms were noted without visible $P$ waves $(n=3)$ or with $P$ waves partially hidden in the QRS complex or T waves $(\mathrm{n}=3)$ (fig 2$)$. The $\mathrm{P}$ waves were positive in the inferior leads (fig 2) indicating normal craneocaudal activation of the atria. This is in contrast with the caudocraneal activation (negative $P$ waves in the inferior leads) in patients with neck palpitations caused by atrioventricular nodal reentrant tachycardia.

\section{ELECTROPHYSIOLOGICAL STUDY}

Five patients underwent an electrophysiological examination where quadripolar electrode catheters were inserted into a femoral vein and positioned in the high right atrium, in the His bundle position, and in the apex of the right ventricle. In four patients, normal baseline atrioventricular conduction intervals were present and in one there was a prolonged $\mathrm{AH}$ interval of $315 \mathrm{~ms}$ at the start of the study (table 1).

In all five patients dual atrioventricular nodal pathways were present. ${ }^{3}$ A large autonomically mediated intrapatient variability in the anterograde refractory period of the fast pathway in combination with long slow pathway conduction times (shortest AH slow pathway 425 (121) ms) explained how a late ventricular activation after slow pathway conduction would (nearly) coincide with the next atrial depolarisation during sinus tachycardia (four patients) or normal sinus rhythm (two patients). Thus simultaneous contraction of atria and ventricles caused neck pounding. Figure 3 shows this in one of the patients: during sinus tachycardia with an RR interval of $440 \mathrm{~ms}$, activation of the ventricles after slow pathway conduction nearly coincided with the next atrial activation.

In none of the patients could an atrioventricular nodal reentrant tachycardia be induced; in four patients no or very poor retrograde ventriculoatrial conduction was present during right ventricular pacing, and in one patient retrograde ventriculoatrial block occurred at a ventricular pacing cycle of $560 \mathrm{~ms}$.

No other supraventricular or ventricular tachyarrhythmias could be induced in any patient with programmed electrical stimulation of the heart.

CLINICAL COURSE

In three patients whose main complaint was palpitations with the slightest exercise a $\beta$
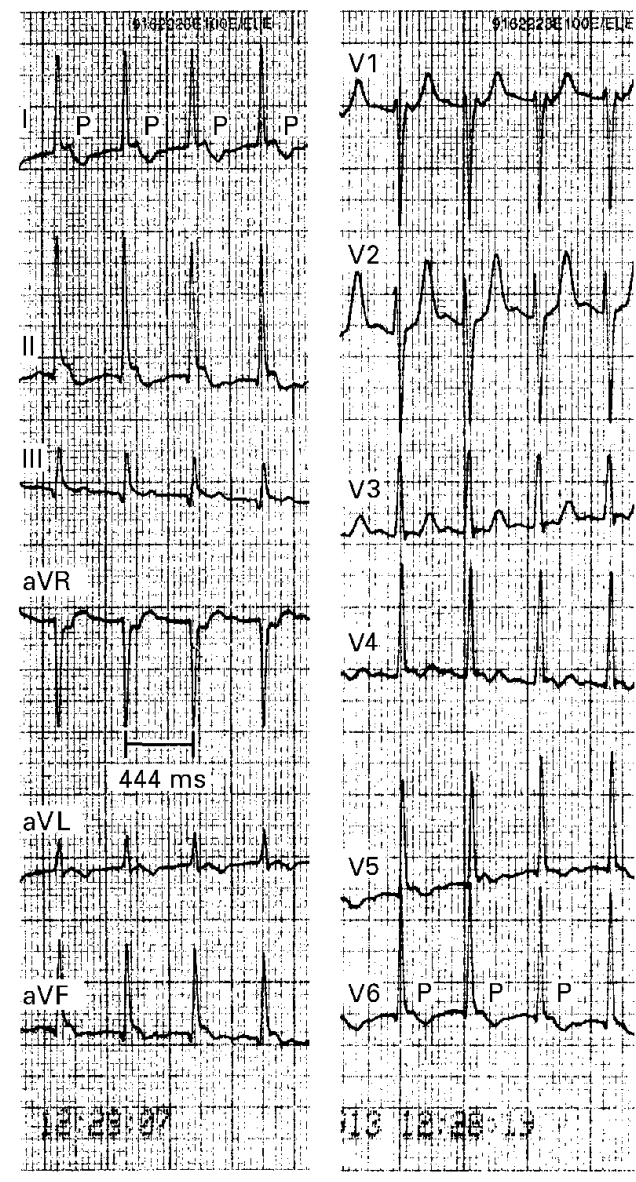

Figure 2 Twelve lead electrocardiogram of patient 2 during complaints of palpitations and neck pounding. $A$ narrow QRS tachycardia of 135 beats/min is seen. P waves that are partially hidden in the terminal part of the QRS complex are present in most leads. The electrical axis of the $P$ waves is compatible with a sinus node origin. Paper speed $25 \mathrm{~mm} / \mathrm{s}$.

blocking drug was prescribed, followed by a calcium antagonist. However, this treatment led to an increase in symptoms and the drugs had to be stopped. Because of incapacitating symptoms in two of these patients, a dual chamber pacemaker was implanted. At the time of writing they had been free of complaints for one year and six months respectively.

In the other four patients we decided to postpone active treatment after full discussion with the patients. Since antiarrhythmic drugs appear to aggravate the symptoms in such patients, we decided to refrain from medical treatment; the patients were told that a pacemaker could be installed if symptoms became severe. Although all four patients still have episodes of palpitations with neck pounding, none has asked for active treatment after a follow up ranging from two to five years.

\section{Discussion}

The six patients described in this report had paroxysmal palpitations and neck pounding caused by the presence of dual atrioventricular nodal pathways, not by pathological tachyarrhythmias. The complaint of paroxysmal regular palpitations accompanied by distinctive neck pounding in these patients was suggestive 


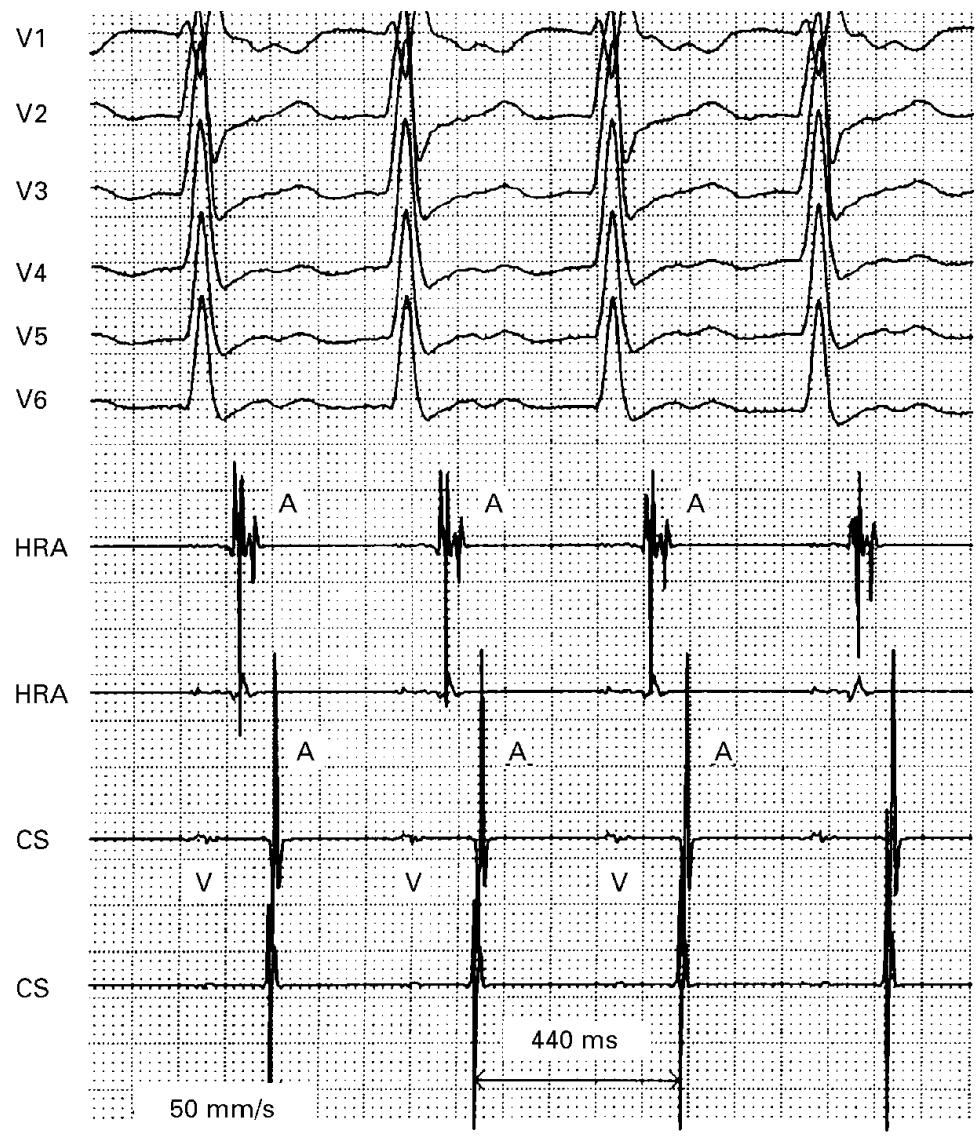

Figure 3 Simultaneous recording of the precordial leads V1 to V6 and bipolar electrograms from the high right atrium (HRA) and the coronary sinus (CS), showing how the atria $(A)$ and ventricles $(V)$ are nearly simultaneously activated during sinus tachycardia at 136 beats/min with atrioventricular conduction over the slow pathway.

of the presence of atrioventricular nodal reentrant tachycardia, although some disparate features were present in the history and sometimes in the electrocardiographic presentation. ${ }^{4}$ In four patients the palpitations mostly occurred on exercise and during emotional disturbance, an association not specially noted in patients with atrioventricular nodal reentrant tachycardia. Two patients complained of prominent heart beats at rest together with neck pounding, but not the fast heart rate typical of atrioventricular nodal reentrant tachycardia.

Electrophysiological studies in these patients showed that the presence of dual atrioventricular nodal pathways was responsible for the neck pounding during sinus rhythm. The long $\mathrm{AH}$ interval during anterograde slow pathway conduction caused the ventricular activation to coincide with the next atrial depolarisation. In four patients this occurred with sinus tachycardia caused by exercise or emotion, and in two the conduction time along the slow pathway was long enough to allow simultaneous activa- tion of the ventricles and atria during normal sinus rhythm at rest. The (nearly) simultaneous activation of ventricles and atria cause atrial contraction against closed atrioventricular valves, leading to jugular reflux and neck pounding. This haemodynamic mechanism of neck pounding has previously been described by our group in patients with atrioventricular nodal reentrant tachycardia. ${ }^{2}$

As recently discussed by Fisch et al, electrocardiographic manifestations of dual atrioventricular nodal pathways other than atrioventricular node reentrant tachycardia are relatively uncommon. ${ }^{5}$ Sudden and persistent prolongation or shortening of the PR interval, PR interval alternans, and dual ventricular responses to a single supraventricular impulse are described. That persistent slow pathway conduction during sinus rhythm can cause palpitations and neck pounding has, to our knowledge, never been reported.

ECG recordings made while our patients are symptomatic might be indistinguishable from atrioventricular nodal reentrant tachycardia if the $\mathrm{P}$ waves are completely hidden in the QRS complex. ${ }^{6}$ If the $P$ waves are partially seen, however, the sinus $\mathrm{P}$ wave morphology can be distinguished and the diagnosis of atrioventricular nodal reentrant tachycardia ruled out.

Therapeutic decision making in these patients turned out to be rather difficult. From knowledge of the underlying electrophysiological disorder, it is understandable that medical treatment could aggravate the symptoms, and this is what we observed in the three patients who received treatment with $\beta$ blockers or calcium antagonists. Implantation of a dual chamber pacemaker relieved the symptoms in two of these patients. In the others this option was discussed. After we had explained the nature of their palpitations, all these patients decided that their complaints did not at present justify the implantation of a pacemaker.

We conclude that neck pounding during sinus rhythm is a clinical manifestation of dual atrioventricular nodal pathways. Medical treatment tends to aggravate symptoms, while a pacemaker may offer definitive relief of symptoms.

1 Brugada P, Gursoy S, Brugada J, et al. Investigation of palpitations. Lancet 1993;342:1254-8.

2 Gursoy S, Steurer G, Brugada J, et al. The hemodynamic mechanism of pounding in the neck in atrioventricular reentrant tachycardia. N Engl f Med 1992;327:772-4.

3 Wellens HJJ, Durrer D. The role of an accessory atrioventricular pathway in reciprocal tachycardia. Circulation $1975 ; 52: 58-72$.

4 Josephson ME, Wellens HJJ. Differential diagnosis of supraventricular tachycardia. Cardiol Clin 1990;8:411-42.

5 Fisch C, Mandrola JM, Rardon DP. Electrocardiographic manifestations of dual atrioventricular node conduction during sinus rhythm. I Am Coll Cardiol 1997;29:1015-22.

6 Elvas L, Gursoy S, Brugada J, et al. Atrioventricular nodal

6 Elvas L, Gursoy S, Brugada J, et al. Atrioventricular nodal
reentrant tachycardia: a review. Can f Cardiol 1994;10: reentrant
$342-8$ 